\title{
Performance of Cognitive Radio Sensor Networks Using Hybrid Automatic Repeat ReQuest: Stop-and-Wait
}

\author{
Fazlullah Khan ${ }^{1}$ - Ateeq ur Rehman ${ }^{1} \cdot$ Muhammad Usman $^{2} \cdot{\text { Zhiyuan } \operatorname{Tan}^{3} \text { - Deepak Puthal }}^{2}$ \\ (c) Springer Science+Business Media, LLC, part of Springer Nature 2018
}

\begin{abstract}
The enormous developments in the field of wireless communication technologies have made the unlicensed spectrum bands crowded, resulting uncontrolled interference to the traditional wireless network applications. On the other hand, licensed spectrum bands are almost completely allocated to the licensed users also known as Primary users (PUs). This dilemma became a blackhole for the upcoming innovative wireless network applications. To mitigate this problem, the cognitive radio (CR) concept emerges as a promising solution for reducing the spectrum scarcity issue. The CR network is a low cost solution for efficient utilization of the spectrum by allowing secondary users (SUs) to exploit the unoccupied licensed spectrum. In this paper, we model the PU's utilization activity by a two-state Discrete-Time-Markov Chain (DTMC) (i.e., Free and busy states), for identifying the temporarily unoccupied spectrum bands,. Furthermore, we propose a Cognitive Radio Sense-and-Wait assisted HARQ scheme, which enables the Cluster Head $(\mathrm{CH})$ to perform sensing operation for the sake of determining the PU's activity. Once the channel is found in free state, the $\mathrm{CH}$ advertise control signals to the member nodes for data transmission relying on Stop-and-Wait Hybrid- Automatic Repeat-Request (SW-HARQ). By contrast, when the channel is occupied by the PU, the $\mathrm{CH}$ waits and start sensing again. Additionally, the proposed CRSW assisted HARQ scheme is analytical modeled, based on which the closed-form expressions are derived both for average block delay and throughput. Finally, the correctness of the closed-form expressions are confirmed by the simulation results. It is also clear from the performance results that the level of PU utilization and the reliability of the PU channel have great influence on the delay and throughput of CRSW assisted HARQ model.
\end{abstract}

Keywords Primary user detection modeling - Cognitive radio sensor networks · Wireless sensor networks $\cdot$ SW-HARQ . CRSW assisted HARQ model

Fazlullah Khan

fazlullah@awkum.edu.pk

Ateeq ur Rehman

ateeq@awkum.edu.pk

Muhammad Usman

muhammad.usman@uts.edu.au

Zhiyuan Tan

Z.Tan@napier.ac.uk

Deepak Puthal

Deepak.Puthal@uts.edu.au

1 Department of Computer Science, Abdul Wali Khan University Mardan, Mardan, KPK, Pakistan

2 Faculty of Engineering and Information Technology, University of Technology Sydney, Ultimo, Australia

3 School of Computing, Edinburgh Napier University, Edinburgh, UK

\section{Introduction}

The $21^{\text {st }}$ century has witnessed exponential growth in innovative wireless applications. These applications have fulfilled the demand of users. However, they have dramatically increased the tele-traffic as well as the usage of electromagnetic spectrum, particularly the sub $-2 \mathrm{GHz}$ frequency bands [1]. The expansion in wireless services leads to the dilemma of spectrum scarcity. To solve the spectrum shortage issue, spectrum regulatory bodies such as Federal Communication Commission (FCC) of the United States (US) and the European Telecommunications Standards Institute (ETSI), have investigated the spectrum utilization in various countries at different time intervals [2-7]. These studies revealed that the electromagnetic spectrum is not physically limited but improperly allocated. The inappropriate 
allocation is due to the static allocation policy, using which the spectrum band is exclusively assigned to the licensed users, also known as primary users (PUs). The PUs are those users who pay for the license and are only authorised to use the assigned spectrum band. For example, the TV stations and cellular users are considered as PUs. The studies of [2-5] have demonstrated that $15-85 \%$ of the licensed spectrum are underutilized due to the current static spectrum allocation policy, resulting in spectrum scarcity. To overcome this dilemma, the concept of dynamic spectrum allocation (DSA) has been proposed which allows the unlicensed users to find the free spectrum, access it and use for data transmission without influencing the legal rights of PUs [2, 8-10]. This technique leads to the emergence of cognitive radio $(\mathrm{CR})$ concept, which is widely accepted for solving spectrum scarcity problems.

The term cognitive radio was first coined by Joseph Mitola in 1999 [11], to solve the problem of spectrum scarcity by efficient utilization of the licensed spectrum. According to [12], the CR as a context-aware intelligent radio has the ability to learn from the environment and dynamically re-configure its transceiver, according to the communication environment. Using these capabilities, the CUs sense the licensed spectrum and transmit their data only when the spectrum band is free from PUs. However, each CU has to vacate the licensed spectrum band upon a PU arrival. The CR concept has been elaborated in [1316]. These capabilities encouraged the regulatory bodies to officially allow the CR concept for maximising spectrum exploitation. In this regard, the phenomenon of $\mathrm{CR}$ has been widely adopted by various wireless standards like IEEE 802:11y, 802:16h, 802:22 and 1900, which has been thoroughly studied in [17].

In literature, various aspects of $\mathrm{CR}$ such as working cycles, designing architecture, spectrum sensing, spectrum sharing, spectrum management, cooperative sensing etc [14, 15, 18-21] have widely been studied. However, limited studies have been conducted in the direction of reliable data transmission. In contrast to conventional wireless systems, in CR systems, the reliability of data transmission is not merely dependant on channel quality but also upon the activity pattern of PUs. Hence, it is highly important to accurately model the PU activity on the channel [2224]. There are numerous work performed on PU and channel modeling. In our previous studies [25-29], we have modelled the activities of PU over the channel using discrete-time-Markov chain (DTMC), in which each state represents the status of a channel. For instance, when the channel is deemed to be free from PUs, it is assumed to be free for $\mathrm{CR}$ and vice versa. Following our previous work, in this paper, we also modelled the PU's channel by two-state Markov chain having free and busy states. To be precise, busy state depicts that the channel is occupied by the PU while free state represents the scenario in which the channel is free from PU and the CR uses it for data transmission.

Secondly, the CR systems face challenges similar to conventional wireless systems, such as noise, interference, fading etc [30, 31]. Apart from these challenges, the CR systems have to face the dynamic activities of PUs, resulting in more complex $\mathrm{CR}$ systems. In this regard, HARQ schemes remained a favourable choice for designing a reliable data transmission scheme. For instance, the authors in [32] studied the performance of HARQ in a third generation partnership project (3GPP) long term evolution (LTE) specification over OFDMA system. D. Nguyen et al. [33] introduced the idea of allowing a transmitter to combine and retransmits the lost packets in such a way that the receiver recovers the packets from a single received copy. Ngo and Hanzo [34] surveyed the HARQ techniques in the context of cooperative wireless communication and a novel relay-switching technique was proposed for enhancing the system's throughput. The HARQ-based techniques are standardized by IEEE, for example, 802.20, 802.16m and 802.16.1 [35-37].

Paucity of studies have take place in the direction of achieving a reliable data transmission in CR systems. For instance, the studies in [38-50] have assumed a reliable data transmission without considering the dynamic activity of the PU and its impact on the CR systems. In this regard, in our previous studies [25-29], we have incorporated various HARQ techniques in CR systems, assuming only a transmitter and a receiver. In contrast to our previous studies, in this paper, we considered an ad hoc CR-based sensor network, comprises of a cluster head $(\mathrm{CH})$ and member nodes. The $\mathrm{CH}$ performs sensing and once it deems a free time-slot (TS), it initiates and broadcasts a clearto-send (CTS) signal to the member nodes and waits for the response. The member nodes respond with a joinrequest and the $\mathrm{CH}$ selects the member node for data transmission, based on first-come-first-serve principle. The selected member node then starts transmitting its data, using a stop-and-wait HARQ approach. On the other hand, when the channel is found to be in busy state, the $\mathrm{CH}$ remains silent and starts sensing again in the subsequent TS. This process continues until a free TS is found.

This paper mainly focuses on the efficient utilization of licensed spectrum by allowing CR-based member nodes to use a PU channel, which results in a higher throughput and a lower delay. We have proposed a CRSW-assisted HARQ model for solving the problem of inefficient utilization of the spectrum. The main contributions of this paper are as follow.

- Designing and modeling a CR-based ad hoc network, assisted by a HARQ scheme for the sake of introducing the cognitive capabilities with the conventional SW-HARQ 
scheme and apply it to sensor network to attain a reliable data delivery.

- The CRSW-assisted HARQ model is analytically modelled using a probabilistic based approach, using which closed-form expressions for average packet delay and throughput are derived.

- Theoretical results of CRSW-assisted HARQ model are validated through simulations using MATLAB. Moreover, the probability distribution, based on end-toend packet delay, is investigated through Monte Carlo simulations.

The remaining paper is organized as follows. In Section 2, the system model is elaborated, with primary user system and assumptions in Section 2.1, whereas Section 2.2 describes modeling of cognitive radio sensor networks. The cognitive radio sense and wait assisted HARQ is discussed in Section 2.3, operation of a cluster head in Section 2.4, and operation of a sensor node in Section 2.5. Analysis of the CRSW assisted HARQ model is discussed in Section 3, delay analysis is performed in Section 3.1, and throughput analysis in Section 3.2, followed by results and discussion in Section 4. Finally, Section 5 elaborates future research scope and concludes the paper.

\section{System model}

In this section, we consider step-by-step description of the system model and assumptions.

\subsection{Primary user system and assumptions}

In this paper, we consider a wireless channel which is exclusively allocated to the PU. For simplicity, we assume the channel is divided into equal length time slots (TS), where each has a duration of $T$ seconds, as illustrated in Fig. 1. In our proposed model, the PU is synchronized to access the channel, based on the TSs. Hence, PUs transmission resemble with the starting and ending of a TS. For example, as depicted in Fig. 1, if a TS is sensed and the activity of the PU is deemed, then PU remains active until the end of TS. However, if a TS is found unoccupied by the PU, then TS remains unoccupied till the end of its duration. With the help of the above procedure, the PU's transmission always occur in the integer multiple of TSs, which means the transmission is always equal to $1,2,3, \ldots$ but not in floating points.

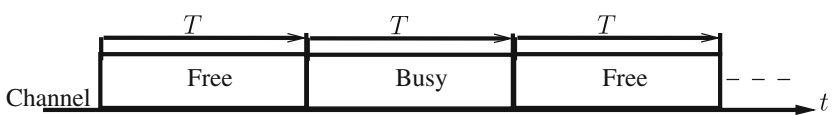

Fig. 1 TS of equal length, Busy or Free period
Moreover, preserving the legal rights of the $\mathrm{PU}$, it can utilize any TS independently with the same probability. Hence, based on these assumptions, the activity of PU over a wireless channel may be modeled by a DiscreteTime Markov Chain (DTMC) having two states (free, busy), where the state to state traversing probabilities are depicted in Fig. 2. Specifically, the 'free' state symbolizes that the PU is inactive, whereas, the 'busy' state shows that the channel is occupied by the PU at the respective TS.

Following the properties of DTMC, a TS could be in any state at time $t$, i.e., $S(t) \in\{f r e e(t)$, busy( $t-$ $1), \ldots$ busy(1)\}. In other words, it can be expressed as

$\mathcal{S}=\left\{S_{1}, S_{2}\right\}$.

where state $S_{1}$ represents a free TS and state $S_{2}$ denotes a busy TS. To determine the state of the TS at time $t$, we have the condition probability that the TS was in state $S_{j}$ at TS $(t+1)$, given that it was in $S_{i}$ in TS $t$, which can be mathematically formulated as

$$
\begin{aligned}
P_{i, j}= & \left\{S(t+1)=S_{j} \mid S(t)=S_{i}, \ldots, S(1)=S_{1}\right\}, \\
= & \left\{S(t+1)=S_{j} \mid S(t)=S_{i}\right\}, \quad \text { where } \\
& i=1,2, j=1,2 \text { and } t=1,2, \ldots
\end{aligned}
$$

Moreover, based on the principles of DTMC, each state must have a probability less than 1 (i.e., $0 \leq P_{i, j} \leq 1$ ) and the outgoing transitions probabilities from state $S_{i}$ should be equal to one $\sum_{j \in S} P(i, j)=1$ [52]. Hence, in our proposed model, we have

$P_{1,1}+P_{1,2}=1$, and $P_{2,2}+P_{2,1}=1$.

The state-to-state transition matrix of two states DTMC is represented by $\mathbf{P}$ and expressed as,

$\boldsymbol{P}=\left[\begin{array}{ll}P_{1,1} & P_{1,2} \\ P_{2,1} & P_{2,2}\end{array}\right]$

where $P_{1,1}$ and $P_{2,2}$ represent the probability of being in same state whereas $P_{1,2}$ and $P_{2,1}$ denotes the traversing of states, i.e., from free to busy state and from busy to

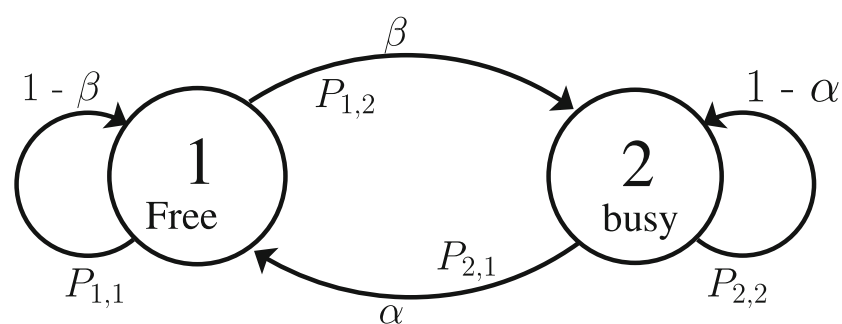

Fig. 2 Two-state DTMC model of the PU system, where $\alpha$ and $\beta$ represents the transition from busy to free and from free to busy state, respectively $[26,51]$ 
free state, respectively, as shown in Fig. 2. Then, when the Markov chain become steady i.e.,

$\boldsymbol{p}(t+1)=\boldsymbol{P}^{T} \boldsymbol{p}(t)$,

$$
\left(\boldsymbol{P}^{T}\right)^{t} \boldsymbol{p}(1) .
$$

where $\boldsymbol{p}(1)$ represents the starting state (i.e., $\boldsymbol{p}(1)=[1,0]$ ). Furthermore, $\boldsymbol{P}^{T}$ is a left stochastic matrix due to the fact that summation of each column is equal to 1 [53], which is also verified in Eq. 3. Hence, when the Markov chain becomes steady, we have,

$\boldsymbol{p}(t+1)=\boldsymbol{p}(t)$.

Moreover, let us symbolize the steady state probabilities by $\pi=\left[\pi_{1}, \pi_{2}\right]$, then we have a recursive equation of [52]

$\pi=P^{T} \pi$

In Eq. $7, \boldsymbol{\pi}$ is the right Eigen vector of the transition metric $\boldsymbol{P}$ with the Eigen value of 1 . It is important to note that the steady state vector satisfies the condition of

$\pi_{1}+\pi_{2}=1$ or $\boldsymbol{\pi}^{T} \mathbf{1}=1$.

where 1 represents a column vector having all 1 values. For the sake of deriving close-form expressions for both states, let $P_{f}$ and $P_{b}$ represent the probability of being in the free state and busy state, then we have,

$P_{b} \alpha=P_{f} \beta$. which reaches to:

$\pi_{1}=\left(\frac{P_{2,1}}{P_{1,2}+P_{2,1}}\right)$ and $\pi_{2}=\left(\frac{P_{1,2}}{P_{1,2}+P_{2,1}}\right)$.

which can also be represented by:

$P_{f}=\frac{\alpha}{\alpha+\beta}$ and $P_{b}=\frac{\beta}{\alpha+\beta}$

Following the above discussion, we may conclude that when a TS is not occupied by the PU, it will remain free for the whole $T$ seconds duration. Thus, for the sake of improving the overall channel utilization, the SU may access the free TSs and transmits its own data packets [9, 54]. In the following subsection, we will discuss in detail the procedure of finding a free TS and data transmission.

\subsection{Modeling the cognitive radio sensor network (CRSN)}

We assume that a CRSN is employed in an area of a 217 PU system, where signal-to-noise ratio is very high. A 218 sensor network has low power, low processing capability, 219 and exposed to noise, so these networks cannot perform 220 well in the presence of high noise and interference in 221 the unlicensed band. In this paper, CRSN consists of one

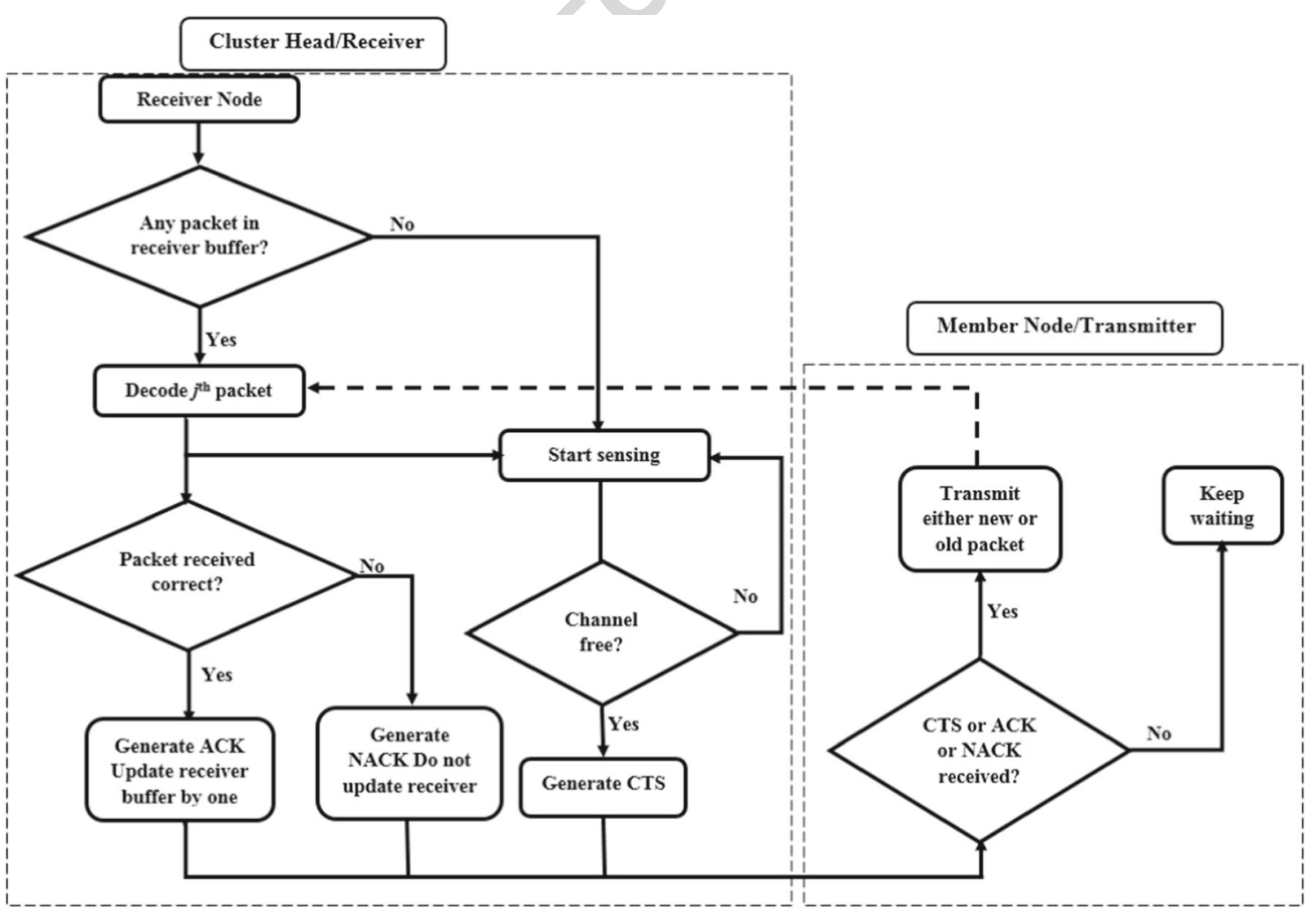

Fig. 3 Flow chart of CRSW assisted HARQ model 
cluster comprise of Cluster Head $(\mathrm{CH})$ and member nodes. The $\mathrm{CH}$ communicates with member nodes, where the member nodes use time division multiple access mechanism for transmitting data packets in a specified time-frame. Specifically, the $\mathrm{CH}$ perform sensing in order to ideally detect the activity (free/busy) of the PU over the channel, regardless of false-alarm and miss-detection. Hence, when a free TS is detected, a member node transmits a packet to the $\mathrm{CH}$. On the other hand, when $\mathrm{CH}$ detects a busy TS, then it waits until a free TS is deemed. The member nodes follows the stop and wait hybrid automatic repeat request (SW-HARQ) approach for data transmission, which will be explained in the following sections.

\subsection{Cognitive radio sense and wait (CRSW) assisted HARQ}

The proposed CRSN perform two vital functions of sensing PU channel and data transmission. For instance, when a free TS is deemed, then the member node uses the classic SW-HARQ approach for the sake of reliably transmitting data packet to the $\mathrm{CH}$. To achieve high reliability, the $\mathrm{CH}$ incorporates Reed-Solomon (RS) encoding/decoding technique, in order to detect and/or rectify errors in the received packet. To do so, we assume that each data packet is encoded with $\mathrm{RS}$ codeword $\mathrm{RS}\left(\mathrm{N}_{d}, \mathrm{~K}_{d}\right)$ [55], where $K_{d}$ is number of information and $N_{d}$ is coded symbol. The propagation time of each coded packet from member node to the $\mathrm{CH}$ is $T_{r}$ seconds, where $T_{r}<T$. The $\mathrm{RS}$ code is assumed to be capable of perfectly detecting errors as well as rectifies $e=\frac{N_{d}-K_{d}}{2}$ error symbols. Hence, when a received packet has more than $e$ errors, it is considered as erroneous which requires retransmission. The round-triptime of a packet is $T$ seconds, which can be defined as, the time duration from the transmission until the reception of its feedback.

Based on the above assumptions, the data is exchanged between member nodes and $\mathrm{CH}$ over PU channel using the principal of CRSW assisted HARQ model depicted in Fig. 3, and explained in Algorithm 1. The detail operations of $\mathrm{CH}$ and member nodes are provided below.

\subsection{Operations of cluster head}

In our proposed model, the $\mathrm{CH}$ performs the following operations. First, it performs sensing operation for the sake of detecting the activity of a PU over the channel. Once it detects the activity of a PU, it will wait for a while and then starts sensing again. This process continues until a free TS is found. Secondly, we assume that the $\mathrm{CH}$ has a finite buffer for storing the index number of packets that is expected to be received. The buffer follows First-in-First-out (FIFO) principle. Thirdly, when a free TS is detected, the $\mathrm{CH}$ broadcasts an Clear-to-Send (CTS) signal to the member nodes and waits for them to respond. We assume that the $\mathrm{CH}$ selects a member node for transmission based on the First-Come-First-Serve (FCFS) principle. In other words, it means that the member node which receives the CTS signal and respond earlier is allowed to transmit new/old packet. It is worth mentioning that each member node acknowledges the CTS signal with a join-request (Readyto-Send) response. When the corresponding member node finishes its transmission, it then starts waiting for the reception of feedback signal from the $\mathrm{CH}$, which will be explained in the following subsection.

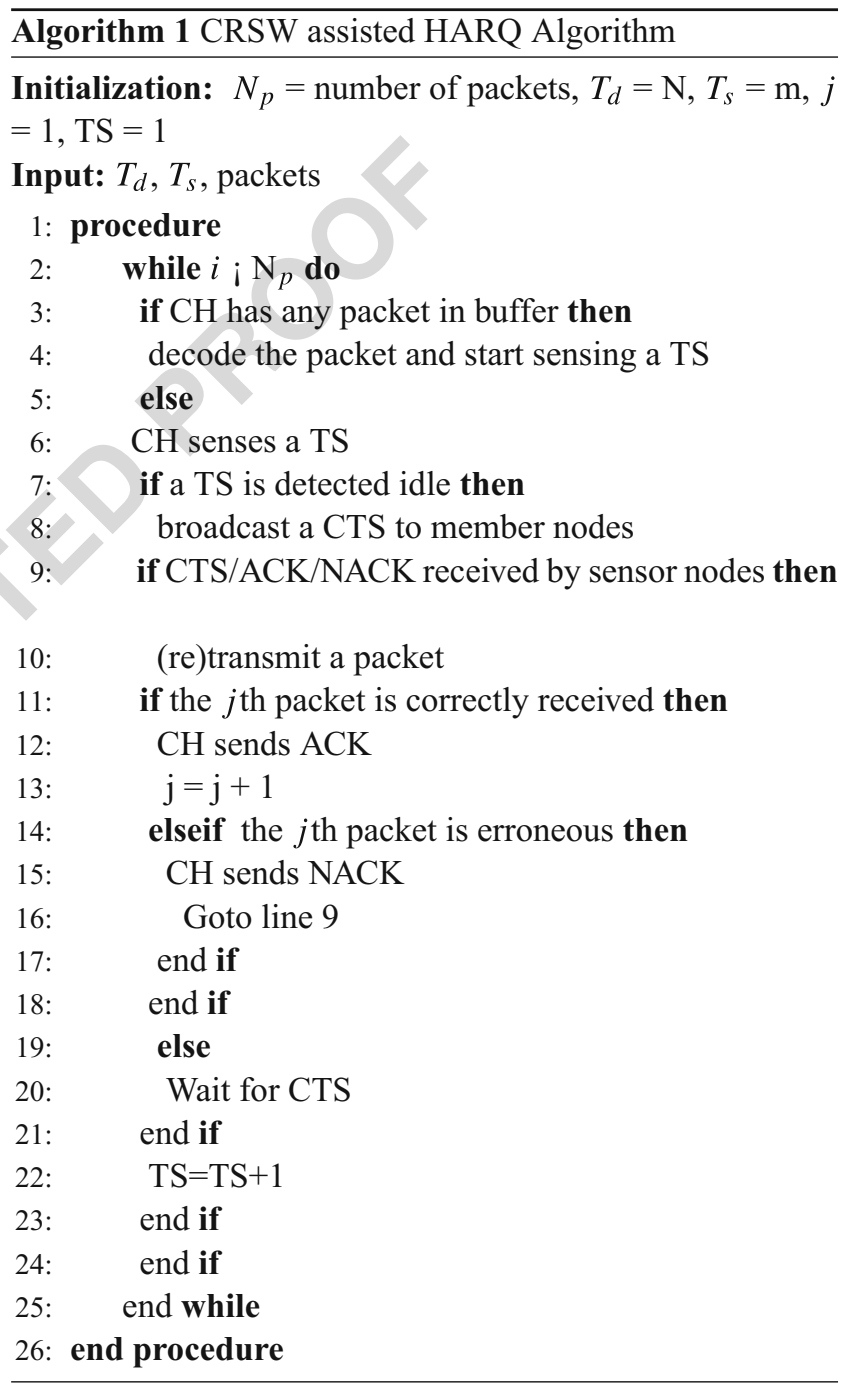

On the other side, when $\mathrm{CH}$ receives a complete $\mathrm{RS}$ coded packet, it performs decoding/correction process in order to generate positive acknowledgment (ACK) or negative acknowledgment (NACK) signal for an error-free or erroneous packet respectively. Specifically, when an error-free packet is received, the $\mathrm{CH}$ increments its buffer index by one and generates immediately an ACK signal and 
transmits to the respective member node. In contrast, when an erroneous packet is received, the buffer index remains unchanged and the $\mathrm{CH}$ responds back with a NACK signal to the member node. This process continues until all packets are correctly received.

\subsection{Operation of member node}

The member nodes of a $\mathrm{CH}$ has a joint buffer in which all the packets are stored in an ascending order. Each member node transmits only one packet at a time from this buffer due to the implementation of SW-HARQ approach. Once a member node receives CTS signal and the $\mathrm{CH}$ authorizes it for packet transmission, it then transmits either new/old packet waiting in the joint buffer. At this time interval, the rest of the member nodes are assumed to remain silent (even if they have responded with an RTS signal) until the advertisement of another CTS signal and this concept is out of the scope of this paper. Furthermore, after the transmission of the $i$ th packet, the corresponding member node waits for $T_{w}$ seconds to receive the feedback of the transmitted packet. Hence, when an ACK is received, the member node deletes the copy of the successfully received packet from the joint buffer and increments its sequence by one. On the other hand, when a NACK signal is received, the corresponding packet remains in the joint buffer with the same index number. Note that the erroneous packet will be retransmitted again by any member node in the next free TS. This process continues until all the packets are correctly transmitted from the member nodes joint buffer.

\section{Analysis of CRSW assisted HARQ model}

In this section, we analyze CRSW assisted HARQ model both in terms of two performance metrics 1) the average block delay and 2) the Average throughput.

\subsection{Average block delay $\left(T_{D}\right)$}

The average block delay can be defined as the average number of TSs or $T_{r}^{\prime} s$ required for the error-free transmission of a packet. In contrast to conventional transmission schemes, in our proposed model, the delay is not only imposed by channel errors, but also comprise of the delay introduced the unavailability of the PU channel for CR transmission. Therefore, let $D_{P}$ represents the delay caused by the utilization the channel by $\mathrm{PU}$ and $D_{e}$ is the delay induced by one or more retransmissions of a packet. In order to analyze the total delay $T_{D}$ of the proposed CRSW assisted HARQ system, we will first investigate the delay induce by the PU activity.
Based on the probability, $P_{b}$ of the PU system defined in Eq. 11, the average delay $D_{P}$ for a $\mathrm{CH}$ to detect a free TS can be calculated as

$$
\begin{aligned}
D_{P} & =E\left[D_{P}(i)\right] \\
& =E[(i-1) T] \\
& =\sum_{i=1}^{\infty}(i-1) T P_{b}^{i-1}\left(1-P_{b}\right) \\
& =\frac{P_{b} T}{1-P_{b}}
\end{aligned}
$$

where $D_{P}(i)$ represents the delay for detecting an $i$ th free TS which can be used by the member node for data transmission, while the prior $(i-1) \mathrm{TS}$ were occupied by PU which causes a delay of $(i-1)$ T. Upon substituting $P_{b}=\frac{\beta}{\alpha+\beta}$ presented in Eq. 11 into Eq. 12, we reach to the following closed-form expression

$T_{D P}=\frac{\beta T}{\alpha}$.

Secondly, when an $i$ th free TS is detected by the $\mathrm{CH}$, it requests the member node for the transmission of a packet which is explained in Sections 2.4 and 2.5. The authorized member node transmit a packet to the $\mathrm{CH}$, however, due to communication impairments, there is a possibility that the packet might be received erroneously, which requires retransmission. The retransmission process continues until the corresponding packet is received without errors. Hence, to accommodate the case of one or more transmissions of a single packet, let us assume that probability of packet being in error is $P_{e}$, then have

$$
\begin{aligned}
D_{e} & =\sum_{i=1}^{\infty}(i) P_{e}^{i-1}\left(1-P_{e}\right) \\
& =\frac{1}{1-P_{e}} .
\end{aligned}
$$

Now the total block delay can be achieved as

$$
\begin{aligned}
T_{D} & =\left(D_{P}+T\right) \times D_{e}, \\
& =\left(\frac{\beta T}{\alpha}+T\right) \times \frac{1}{1-P_{e}}, \\
& =\left(\frac{\alpha+\beta}{\alpha}\right) \times \frac{T}{1-P_{e}} \text { (seconds). }
\end{aligned}
$$

Moreover, the normalized $T_{D}$ in terms of packet transmission $\left(T_{r}\right)$ can be expressed as

$T_{D}=\left(\frac{\alpha+\beta}{\alpha}\right) \times \frac{1}{1-P_{e}} \times \frac{T_{r}+T_{w}}{T_{r}}\left(T_{r}^{\prime} s\right)$.

From Eqs. 15 and 16, we may conceive that the block delay increases with increase in the utilization level of the PU, decrease in the channel quality and with the increase in the waiting time spend for the reception of feedback.

\subsection{Average throughput}

The throughput of the proposed CRSW assisted HARQ model can be defined as the successful transmission rate 
of a packet in TS. The successful transmission of packets depends on the successful detection of free TS by a $\mathrm{CH}$ and error-free transmission of packet to the $\mathrm{CH}$. To investigate throughput of the proposed system, let us represent the probability of successfully delivering a packet to $\mathrm{CH}$ by $P_{S}(i)$ in $i$ th TS, while the remaining $(i-1)$ TSs were either occupied by PU or packet was received in error. Then, $P_{S}(i)$ can be calculated as shown in Eq. 17 below,

$P S(i)=\sum_{j=1}^{i} P_{f}(j \mid i) P_{S}(j \mid i)$,

where the probability that PU channel is free in $j / i$ TS is represented by $P_{f}(j \mid i)$, while $P_{S}(j \mid i)$ is the probability that the member node successfully transmits a packet in $j$ TS. Therefore, we can write Eq. 18 based on Eq. 17 as follow,

$$
\begin{aligned}
P_{S}(i) & =\sum_{j=1}^{i}\left(\begin{array}{l}
i \\
j
\end{array}\right) P_{f}^{j} P_{b}^{(i-j)} P_{e}^{(j-1)}\left(1-P_{e}\right), \\
& =\sum_{j=1}^{i}\left(\begin{array}{l}
i \\
j
\end{array}\right)\left(\frac{\alpha}{\alpha+\beta}\right)^{j}\left(\frac{\beta}{\alpha+\beta}\right)^{(i-j)} P_{e}^{(j-1)}\left(1-P_{e}\right) .
\end{aligned}
$$

Based on the above equation, we can readily obtain the normalized throughput as:

$$
\begin{aligned}
\eta= & \sum_{i=1}^{\infty} \frac{1}{i} \times P_{S}(i), \\
= & {\left[\sum_{i=1}^{\infty} \sum_{j=1}^{i}\left(\begin{array}{l}
i \\
j
\end{array}\right)\left(\frac{\alpha}{\alpha+\beta}\right)^{j}\left(\frac{\beta}{\alpha+\beta}\right)^{(i-j)} \times P_{e}^{(j-1)} \times\right.} \\
& \left.\quad \times\left(1-P_{e}\right)\right](\text { packets per TS }) .
\end{aligned}
$$

Moreover, the normalized throughput in terms of packets per $\mathrm{T}_{r}$ can be expressed as

$\bar{\eta}=\frac{T_{r}}{T} \times \eta=\frac{T_{r}}{T_{r}+T_{w}} \times \eta\left(\right.$ packets per $\left.T_{r}\right)$.

\section{Performance results}

In this section, we evaluate both the delay and throughput performance of the proposed CRSW assisted HARQ scheme in terms of PU channel utilization and channel reliability. We illustrate the effect of packet error probability $\left(P_{e}\right)$ and the probability of a channel being in busy state $\left(P_{b}\right)$ on the performance of the proposed scheme. The CRSW assisted HARQ mode has been build with the help MATLAB, where sixty thousands packets have been transferred for each scenario. The simulation start from the sensing of the first TS and ends on the successful reception of total $N_{s}$ packets.

Figure 4 depicts the throughput achieved by CRSW assisted HARQ model against $P_{e}$ and $P_{b}$ of the channel.

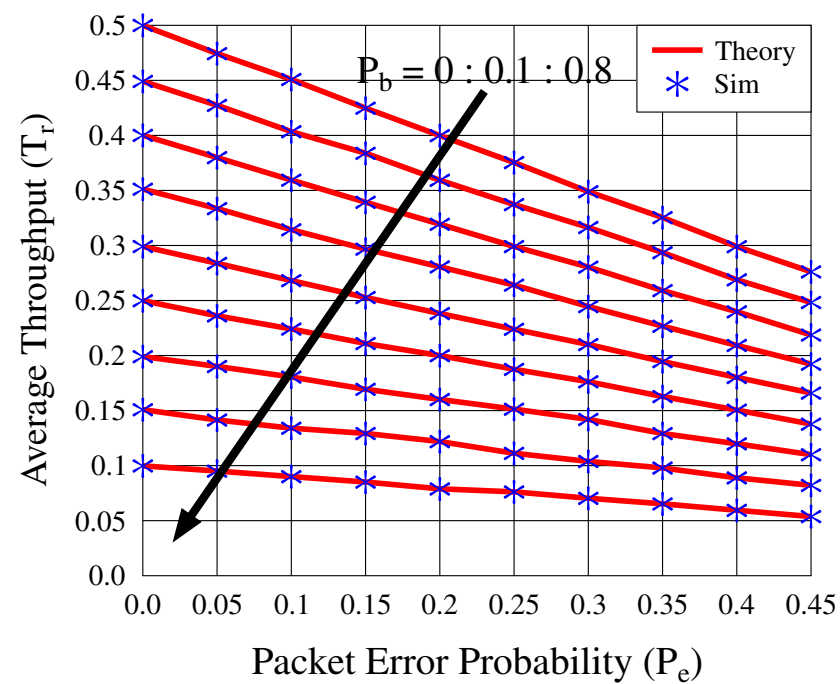

Fig. 4 Throughput versus packet error probability $P_{e}$ for the CRSW assisted HARQ model related with different $P_{b}$, when presuming $T_{r}=$ 1 and $T_{w}=1$ seconds. Average throughput performance is examined for different values of $P_{e}$, where results are calculated in terms of $T_{r}$

The formula used for calculating the throughput is given in Eq. 21 as

$\eta_{S}^{\prime}=\frac{N_{s}}{N_{t s}} \cdot \frac{T_{r}}{T_{r}+T_{w}}$ (packets per $\left.T_{r}\right)$,

where $N_{s}$ is the sum of packets correctly transmitted by the member nodes in the total $N_{t s}$ TSs.

We can see in Fig. 4 that the throughput is at its peak for $P_{e}=0$, this means that the channel is highly reliable and therefore, the rate of retransmission is minimum. However, as shown in Fig. 4, the throughput drops when $P_{e}$ increases. This is because, the channel reliability reduces which results in low throughput. The decrease in throughput is almost linear, this is due retransmission of packets. For certain $P_{e}$, the throughput reaches to its maximum level, when there is no PU activity on the channel, i.e. when $P_{b}=0$. But when, $P_{b}$ increases, the achievable throughput considerably decreases, as the $\mathrm{CH}$ has to wait longer duration for detecting free TSs. For instance, both when $P_{e}$ and $P_{b}$ are zero, then the throughput is at its maximum with the value of .5 seconds. This means that the channel is always free from PU and packet transmission is always successful in the first TS. Furthermore, it is pretty clear form Fig. 4 that the analytical results calculated from Eq. 20 agree well with our simulation results.

After investigating the throughput performance, we now explain on the delay of the CRSW assisted HARQ model. First we study average block delay, as presented in Fig. 5. It is worth mentioning here that for simulation results, we have calculated the average packet delay using Eq. 22

$T_{D S}=\frac{N_{t s} \cdot\left(T_{r}+T_{w}\right)}{N_{s}}$ (seconds). 


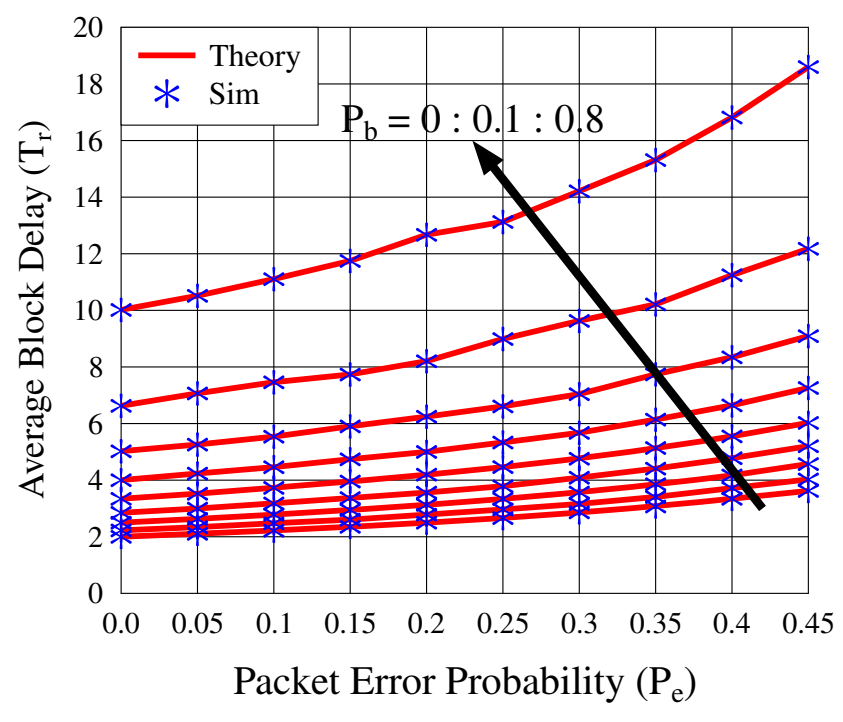

Fig. 5 Average block delay of the CRSW assisted HARQ model against probability of packet error for different $\mathrm{P}_{b}$ values where $N_{s}$ is the sum of packets correctly transmitted by member nodes in the total number of TSs $N_{t s}$. The results shown in Fig. 5 are normalized by $T_{r}$, producing Eq. 23

$T_{D S}^{\prime}=\frac{T_{D S}}{T_{r}} \quad\left(T_{r} s\right)$.

Figure 5, depicts the average packet delay of the CRSW assisted HARQ model. For certain $P_{b}$, the average packet delay reaches to a minimum level, particularly when the channel is reliable, i.e. when $P_{e}=0$. But when, $P_{b}$ and/or $P_{e}$ increases, the average packet delay considerably

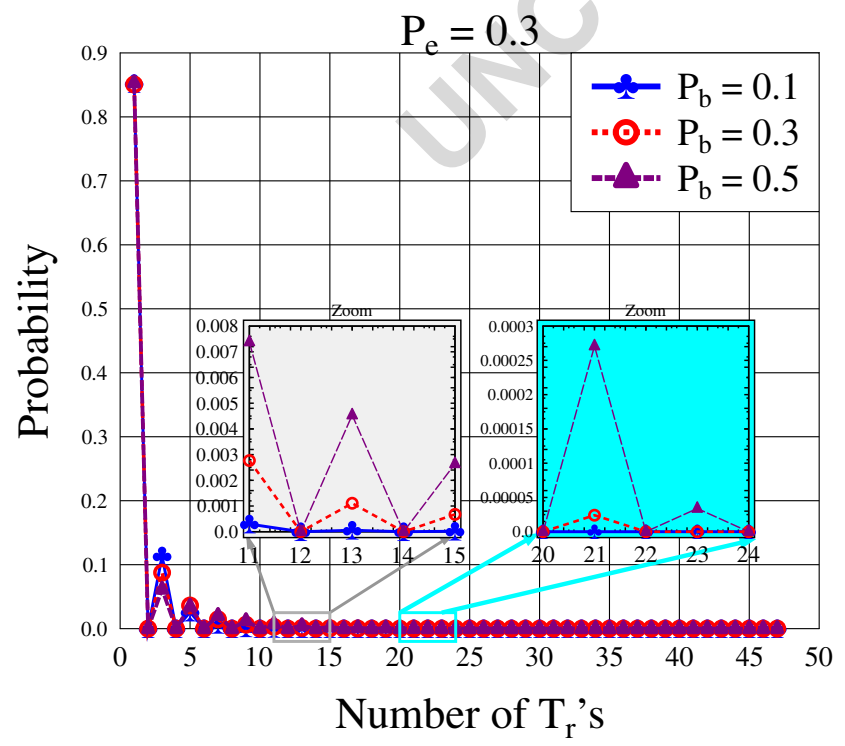

Fig. 6 Characterizing the probability distribution of the E2E packet delay for $P_{b}=\{0.1,0.3,0.5\}$ and $P_{e}=\{0.3\}$

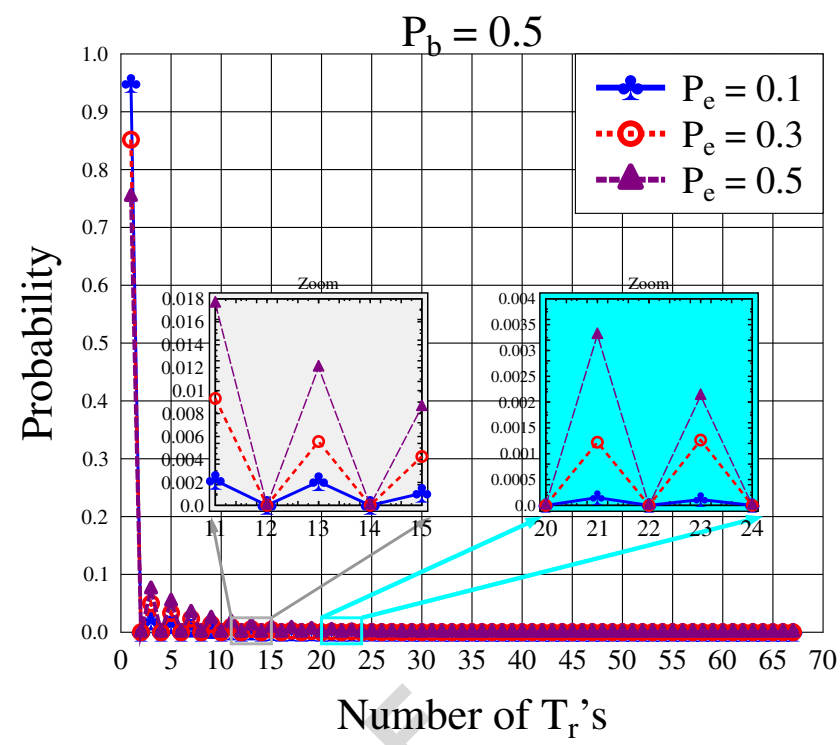

Fig. 7 Investigation of probability distribution of the E2E packet delay for $P_{e}=\{0.1,0.3,0.5\}$ when $P_{b}=\{0.5\}$

increases. This increase in average packet delay is due to the fact that the high value $P_{e}$ causes more retransmissions while increase in $P_{b}$ value decreases the chances of transmission for a member node. Furthermore, it is pretty clear form Fig. 5 that the analytical results calculated from Eq. 16 agree well with our simulation results.

In Fig. 6, we illustrate the end-to-end (E2E) packet delay of the CRSW assisted HARQ model. In our simulations, the E2E delay is defined as the time taken by a packet from its first transmission attempt to its final successful reception divided by total total number of packet.

For E2E, we consider a vector $\boldsymbol{v}$ having length of $N_{s}$ for storing E2E delay of each transmitted packet; then let $\boldsymbol{v}(i)$ denotes the E2E delay of the $i$ th packet, then the probability distribution $\left(P_{d}\right)$ of E2E packet delay shown in Fig. 6 may be calculated as:

$\boldsymbol{P}_{d}=\frac{\sum_{i=1}^{N_{s}} \delta(v(i)-n)}{N_{s}}, 1 \leq n \leq \max (\boldsymbol{v})$.

where $\delta$ function is used for finding the number of TS took by each packet. For example, if 100 packets are successfully received in their first transmission attempt in and 80 packets have taken two $T_{r} s$ then the distribution becomes $\boldsymbol{P}_{d}=$ $\left[100 / N_{s}, 80 / N_{s}, \ldots\right]$ (Fig. 7).

\section{Conclusion}

The latest trends in wireless communication technologies have given the notion that the spectrum is scarce. The spectrum scarcity is due to the static allocation of spectrum, 
and to overcome this issue we need a perfect PU detection model. In this paper, we have proposed and examined the performance of CRSW assisted HARQ model for efficient detection the PU channel. The throughput and delay of CRSW assisted HARQ model has been analyzed mathematically and validated via simulations. Accuracy of the derived closed-form expressions of CRSW assisted HARQ model has been verified using MATLAB simulation. We conclude from simulation results that delay and throughput performances of CRSW assisted HARQ model are mainly affected by both the activities of the PU as well as by the reliability of the PU channel used by SU. When the PU channel is not free, this causes low throughput of the CRSW assisted HARQ model and higher delay, though the channel might be reliable, i.e., $P_{e}=0$. In future research we aim to evaluate the performance of CRSW assisted HARQ model in a realistic imperfect sensing scenarios and multi-cluster scenarios.

\section{References}

1. Goldsmith A, Jafar SA, Maric I, Srinivasa S (2009) Breaking spectrum gridlock with cognitive radios: an information theoretic perspective. Proc IEEE 97(5):894-914

2. FCC (2002) Et docket No. 02-155 spectrum policy task force report technical report

3. Staple G, Werbach K (2004) The end of spectrum scarcity [spectrum allocation and utilization]. IEEE Spectr 41(3):48-52

4. Yang J (2005) Spatial channel characterization for cognitive radios, Master's Thesis, EECS Department, University of California, Berkeley, Tech. Rep. UCB/ERL M05/8. [Online]. Available: http://www.eecs.berkeley.edu/Pubs/TechRpts/2005/4293.html

5. McHenry MA, Tenhula PA, McCloskey D, Roberson DA, Hood CS (2006) Chicago spectrum occupancy measurements \& analysis and a long-term studies proposal. In: Proceedings of the first international workshop on technology and policy for accessing spectrum (TAPAS)

6. Cabric D (2007) Phd thesis on cognitive radios: system design perspective. University of California at Berkeley

7. Islam MH, Koh CL, Oh SW, Qing X, Lai YY, Wang C, Liang YC, Toh BE, Chin F, Tan GL, Toh W (2008) Spectrum survey in singapore: occupancy measurements and analyses. In: 3rd International conference on cognitive radio oriented wireless networks and communications (CrownCom), pp 1-7

8. Hossain E, Niyato D, Han Z (2009) Dynamic spectrum access and management in cognitive radio networks. Cambridge University Press

9. Zhao Q, Sadler BM (2007) A survey of dynamic spectrum access. IEEE Signal Process Mag 24(3):79-89

10. Akhtar F, Rehmani MH, Reisslein M (2016) White space: definitional perspectives and their role in exploiting spectrum opportunities. Telecommun Policy 40(4):319-331

11. Mitola J, Maguire GQ (1999) Cognitive radio: making software radios more personal. IEEE Pers Commun 6(4):13-18

12. Haykin S (2005) Cognitive radio: brain-empowered wireless communications. IEEE J Select Areas Commun 23(2):201-220

13. Cabric D, Mishra SM, Brodersen RW (2004) Implementation issues in spectrum sensing for cognitive radios. In: Conference on signals, systems and computers, conference record of the thirty-eighth asilomar, vol 1, pp 772-776
14. Ganesan G, Li Y (2007) Cooperative spectrum sensing in cognitive radio, part I: two user networks. IEEE Trans Wirel Commun 6(6):2204-2213

15. Yucek T, Arslan H (2009) A survey of spectrum sensing algorithms for cognitive radio applications. IEEE Commun Surv Tutor 11(1):116-130. Quarter

16. Axell E, Leus G, Larsson EG, Poor HV (2012) Spectrum sensing for cognitive radio: state-of-the-art and recent advances. IEEE Signal Process Mag 29(3):101-116

17. IEEE recommended practice for information technologytelecommunications and information exchange between systems wireless regional area networks (WRAN)-specific requirementspart 22.2: installation and deployment of IEEE 802.22 systems," IEEE Std 802.22.2-2012, pp 1-44, Sept 2012

18. Akyildiz IF, Lee W-Y, Vuran MC, Mohanty S (2006) Next generation/dynamic spectrum access/cognitive radio wireless networks: a survey. Comput Netw J (Elsevier) 50(13):2127-2159

19. He A, Gaeddert J, Bae KK, Newman TR, Reed JH, Morales L, Park C-H (2009) Development of a case-based reasoning cognitive engine for IEEE 802.22 WRAN applications. ACM SIGMOBILE Mobile Comput Commun Rev 13(2):37-48

20. Liang YC, Zeng Y, Peh ECY, Hoang AT (2008) Sensingthroughput tradeoff for cognitive radio networks. IEEE Trans Wirel Commun 7(4):1326-1337

21. Khan F, Nakagawa K (2013) Comparative study of spectrum sensing techniques in cognitive radio networks. In: World Congress on computer and information technology (WCCIT) 2013, pp 1-8

22. Su H, Zhang X (2008) Cross-layer based opportunistic MAC protocols for QoS provisionings over cognitive radio wireless networks. IEEE J Selected Areas Commun 26(1):118-129

23. Lee W-Y, Akyildiz IF (2008) Optimal spectrum sensing framework for cognitive radio networks. IEEE Trans Wirel Commun 7(10):3845-3857

24. Akin S, Gursoy MC (2011) Performance analysis of cognitive radio systems under qos constraints and channel uncertainty. IEEE Trans Wirel Commun 10(9):2883-2895

25. Rehman AU, Thomas VA, Yang LL, Hanzo L (2016) Performance of cognitive selective-repeat hybrid automatic repeat request. IEEE Access 4:9828-9846

26. Rehman AU, Dong C, Yang LL, Hanzo L (2016) Performance of cognitive stop-and-wait hybrid automatic repeat request in the face of imperfect sensing. IEEE Access 4:5489-5508

27. Rehman AU, Dong C, Thomas V, Yang LL, Hanzo L (2016) Throughput and delay analysis of cognitive go-back-n hybrid automatic repeat request using discrete-time markov modelling. IEEE Access 4:9659-9680

28. Rehman AU, Yang LL, Hanzo L (2017) Delay and throughput analysis of cognitive go-back-n harq in the face of imperfect sensing. IEEE Access 4

29. Khan F, Rehman AU, Jan MA, Alam M (2017) Modeling resource allocation for real time traffic in cognitive radio sensor networks. In: International conference on future intelligent vehicular technologies, pp 1-8

30. Lin S, Costello DJ (1999) Error control coding: fundamentals and applications, 2nd edn. Prentice-Hall, Upper Saddle River

31. Hanzo L, Liew T, Yeap B, Tee R, Ng SX (2011) Turbo coding, turbo equalisation and space-time coding. EXIT-chart-aided nearcapacity designs for wireless channels, 2nd edn. Wiley

32. Beh KC, Doufexi A, Armour S (2007) Performance evaluation of hybrid ARQ schemes of 3GPP LTE OFDMA system. In: IEEE 18th International symposium on personal, indoor and mobile radio communications, pp $1-5$

33. Nguyen D, Tran T, Nguyen T, Bose B (2009) Wireless broadcast using network coding. IEEE Trans Veh Technol 58(2):914-925 
34. Ngo HA, Hanzo L (2014) Hybrid automatic-repeat-request systems for cooperative wireless communications. IEEE Commun Surv Tutor 16(1):25-45. First

35. IEEE standard for local and metropolitan area networks part 20: Air interface for mobile broadband wireless access systems supporting vehicular mobilityphysical and media access control layer specification, IEEE Std 802.20-2008, pp 1-1039, 2008

36. IEEE standard for local and metropolitan area networks part 16: Air interface for broadband wireless access systems amendment 3: Advanced air interface," IEEE Std 802.16m-2011(Amendment to IEEE Std 802.16-2009), pp 1-1112, 2011

37. IEEE standard for wireless man-advanced air interface for broadband wireless access systems, IEEE Std 802.16.1-2012, pp 1-1090, 2012

38. Li JCF, Zhang W, Nosratinia A, Yuan J (2013) SHARP: spectrum harvesting with ARQ retransmission and probing in cognitive radio. IEEE Trans Commun 61(3):951-960

39. Hamza D, Aissa S (2014) Enhanced primary and secondary performance through cognitive relaying and leveraging primary feedback. IEEE Trans Veh Technol 63(5):2236-2247

40. Harsini JS, Zorzi M (2014) Transmission strategy design in cognitive radio systems with primary ARQ control and QoS provisioning. IEEE Trans Commun 62(6):1790-1802

41. Ao WC, Chen KC (2010) End-to-end HARQ in cognitive radio networks. In: IEEE Wireless communications and networking conference (WCNC), pp 1-6

42. Touati S, Boujemaa H, Abed N (2013) Cooperative ARQ protocols for underlay cognitive radio networks. In: Proceedings of the 21st European signal processing conference (EUSIPCO), pp 1-5

43. Yue G, Wang X, Madihian M (2007) Design of anti-jamming coding for cognitive radio. In: IEEE Global telecommunications conference 2007, pp 4190-4194
44. Yue G, Wang X (2009) Design of efficient ARQ, schemes with anti-jamming coding for cognitive radios. In: IEEE Wireless communications and networking conference (WCNC), pp 1-6

45. Liu Y, Feng Z, Zhang P (2010) A novel ARQ, scheme based on network coding theory in cognitive radio networks. In: IEEE International conference on wireless information technology and systems (ICWITS), pp 1-4

46. Liang W, Ng SX, Feng J, Hanzo L (2014) Pragmatic distributed algorithm for spectral access in cooperative cognitive radio networks. IEEE Trans Commun 62(4):1188-1200

47. Hu J, Yang LL, Hanzo L (2013) Maximum average service rate and optimal queue scheduling of delay-constrained hybrid cognitive radio in nakagami fading channels. IEEE Trans Veh Technol 62(5):2220-2229

48. Makki B, Amat AGI, Eriksson T (2012) HARQ feedback in spectrum sharing networks. IEEE Commun Lett 16(9):1337-1340

49. Makki B, Svensson T, Zorzi M (2015) Finite block-length analysis of spectrum sharing networks using rate adaptation. IEEE Trans Commun 63(8):2823-2835

50. Liang W, Nguyen HV, Ng SX, Hanzo L (2016) Adaptive-TTCMaided near-instantaneously adaptive dynamic network coding for cooperative cognitive radio networks. IEEE Trans Veh Technol 65(3):1314-1325

51. Hong X, Wang J, Wang CX, Shi J (2014) Cognitive radio in $5 \mathrm{~g}$ : a perspective on energy-spectral efficiency trade-off. IEEE Commun Mag 52(7):46-53

52. Bertsekas D, Gallagher R (1991) Data networks, 2nd edn. Prentice Hall

53. Howard R (1971) Dynamic probabilistic systems: Markov models. Wiley, New York

54. Ozcan G, Gursoy MC (2013) Throughput of cognitive radio systems with finite blocklength codes. IEEE J Selected Areas Commun 31(11):2541-2554

55. Lin S, Costello DJ Jr (1999) Error control coding fundamentals and applications, 2nd edn. Prentice-Hall, NJ 